\title{
A 55-year-old man with mild shortness of breath
}

\author{
A. D. Egorova · J. M. Smit • P. Kiès
}

Published online: 23 July 2019

(C) The Author(s) 2019

\section{Answer}

The correct answer is $\mathrm{C}$.

The chest radiograph shown in Fig. 1a of the Question shows the silhouette of a right-sided aortic arch coursing to the right of the trachea. This is a rare, often asymptomatic and incidental finding in the setting of an otherwise structurally normal heart, which was first described in 1763 [1]. It is estimated to occur in $0.04-0.1 \%$ of the population. About half of the cases are associated with an aberrant left subclavian artery, as is illustrated in our patient (type II right-sided aortic arch variant). Other types of right-sided aortic arch variants include mirror-image arch branches (type I) and an isolated left subclavian artery communicating with the pulmonary artery (type III). Type I right aortic arch in particular is strongly associated with congenital heart disease, most commonly tetralogy of Fallot.

There is no evidence of acute aortic pathology (associated with a second 'false' lumen and dissection flap in the aortic wall), left persistent vena cava superior (associated with a dilated coronary sinus) or a double aortic arch on the images presented. Therefore, we can exclude these options.

A three-dimensional reconstruction of the CT scan (Fig. 1a,b) facilitates our comprehension of the anatomy of the right-sided aortic arch giving rise to a left and right common carotid artery, a right subclavian artery and an aberrant left subclavian artery. The left subclavian artery originates from a vascular pouch, referred to as the Kommerell diverticulum (alternatively called remnant diverticulum or lusoria root) posterior to the trachea and oesophagus, poten- tially compressing these structures. Its aneurysmatic enlargement can lead to dissection and rupture, making it an important clinical phenomenon to recognise [2]. CT and MRI are essential tools for evaluation of this pathology. The timing of the (often complex surgical) management of this congenital pathology remains a matter of debate.

Conflict of interest A.D. Egorova, J.M. Smit and P. Kiès declare that they have no competing interests.

Open Access This article is distributed under the terms of the Creative Commons Attribution 4.0 International License (http://creativecommons.org/licenses/by/4.0/), which permits unrestricted use, distribution, and reproduction in any medium, provided you give appropriate credit to the original author(s) and the source, provide a link to the Creative Commons license, and indicate if changes were made.

\section{References}

1. Fioratti F, Aglietti F. A case of human right sided aorta. Anat Rec. 1763;45:365.

2. Cina CS, Althani H, Pasenau J, et al. Kommerell's diverticulum and right sided aortic arch: a cohort study and review of the literature. JVasc Surg. 2004;39:131-9.

\author{
A. D. Egorova $(\varangle) \cdot$ J. M. Smit · P. Kiès \\ Department of Cardiology, Heart Lung Centre, Leiden \\ University Medical Centre, Leiden, The Netherlands \\ a.egorova@lumc.nl
}



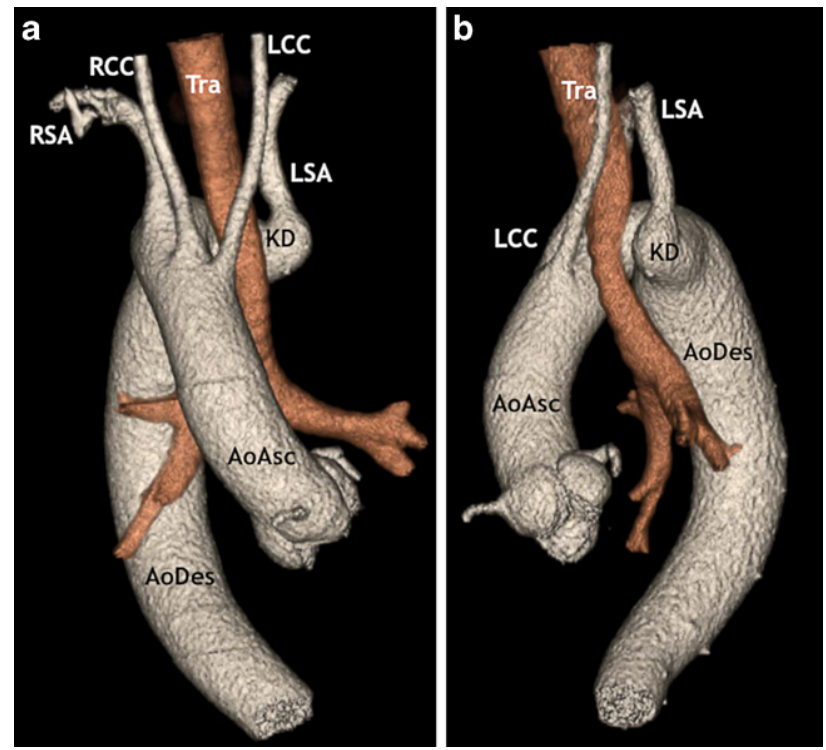

Fig. 1 A three-dimensional reconstruction of the CT angiography showing the frontal view (a) and the left lateral view (b) of the right-sided aortic arch giving rise to the aberrant left subclavian artery from the Kommerell diverticulum. (AoAsc ascending aorta, AoDes descending aorta, Tra trachea, LCC left common carotid artery, RCC right common carotid artery, $R S A$ right subclavian artery, $L S A$ aberrant left subclavian artery, $K D$ Kommerell diverticulum) 